\title{
Failed propofol sedation in a patient with somniloquy - a case report
}

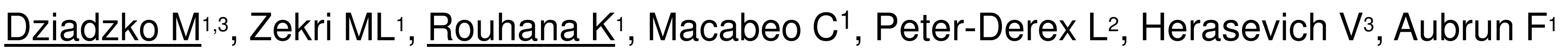
1 - Department of Anesthesiology 2 - Department of Sleep Medicine, CHU Croix Rousse, Lyon, France;

3 - Department of Anesthesiology, Mayo Clinic, Rochester MN, USA

Background: Failed propofol sedation in the outpatient setting is well known in patients with challenging behavior ${ }^{1}$, addiction, or complex medication history. However, some cases remain puzzling.

Case report: ASA I, BMI $19 \mathrm{~kg} / \mathrm{m}^{2}$, age 27 years woman was scheduled for an elective outpatient meniscal repair. Noteworthy medical history included noncomplicated vesicoureteral surgery, and cranial trauma with skull fracture at the age of 10 with no consequences. Only medication was oral contraception. No addictive habits were reported. High level of anxiety was noted at pre-anesthesia evaluation.

One hour prior to surgery, $0.25 \mathrm{mg}$ of alprazolam was given orally. Upon arrival to the OR, the patient was emotionally unstable. Spinal anesthesia was successfully given. However, the patient felt highly uncomfortable at the beginning of the surgery. Propofol TCl (Schindler model) was started with initial target of 0.6 $\mathrm{mg} / \mathrm{ml}$. This led to disruptive comments with no agitation or movements. The target concentration of anesthetic was rapidly increased up to $4 \mu \mathrm{g} / \mathrm{ml}$ with additional IV boluses. Yet, the patient continued to comment on the procedure and environment. We ceased increase of propofol doses as it led to apnea. Natural airways were preserved; the patient kept talking. The surgery was successfully done. The received dose of propofol was $400 \mathrm{mg} / 49 \mathrm{~min}$ (average rate 0.13 $\mathrm{mg} / \mathrm{kg} / \mathrm{min}$ ). Upon arrival in the PACU, the patient was fully awake with no recall of these intraoperative events.
Subsequent interrogation revealed the existence of parasomnia, including somniloquy, bruxism, and periodic limb movements since her childhood. Sleepmedicine specialists were never consulted. 4 hours later, she met criteria for outpatient surgery and was discharged.

Discussion: Parasomnias are frequent in the general population (4\%) and are not always questioned during preanesthesia evaluation. Slow wave sleep parasomnia includes various behaviors and vocalizations resulting from local dissociation of vigilance stages between high-order and primary cortices ${ }^{2}$. Little evidence of anesthesia considerations in parasomnias exists ${ }^{3}$, except those associated with specific conditions ${ }^{4}$ (ex Tourette syndrome). It may be hypothesized that predisposed (parasomniac) patients could exhibit an heterogeneity in cortical activity under general anesthesia leading to motor manifestations without consciousness and memory. This can affect the judgment of the anesthesia efficiency, raising safety concerns from unnecessarily increased doses of sedative drugs. Clinical implications-including dosing of hypnotics, validity of BIS findings, and probable memorization or false memory construction-should be considered and investigated.

\section{Further steps:}

Retrospective study of the association of parasomnia and the increased needs in sedation during anesthesia procedures

Study of EEG patterns in parasomnia patients during the sleep and propofol induction

Anesthesia considerations recommendations for patients with parasomnia 